Ikonion Journal of Mathematics
https://dergipark.org.tr/tr/pub/ikjm
Research Article
Open Access

\title{
An Investigation on The Behaviour of Unbounded Operators in $\Gamma$-Hilbert Space
}

\author{
Sahin Injamamul Islam ${ }^{1}$ (), Nirmal Sarkar ${ }^{\circledR}{ }^{\circledR}$, Ashoke Das $^{3} \oplus$
}

\author{
Keywords: \\ $\Gamma$-Hilbert space, Closed \\ operator, Densely \\ defined operator, Self- \\ adjoint of densely \\ defined operator, \\ Symmetric of densely \\ defined operator.
}

\begin{abstract}
In this paper, we investigate about the behavior of unbounded operators in $\Gamma$-Hilbert Space. Here we discussed about the adjoint, self-adjoint, symmetric and other related properties of densely defined operator. We proof some related theorems and corollaries and will show the characterizations of these operators in $\Gamma$-Hilbert space.
\end{abstract}

Subject Classification (2020): 46CXX, 46C05, 46C07,46C15,46C99,47L06.

\section{Introduction}

$\Gamma$-Hilbert space plays an important role in generalization of general linear quadratic control problems in an abstract space [1] which was motivated from the work of L.Debnath and Pitor Mikusinski [8] but there not enough literature found to study about the unbounded operators in $\Gamma$-Hilbert space. The definition of $\Gamma$-Hilbert space was introduced by Bhattacharya D.K. and T.E. Aman in their paper " $Г$ Hilbert space and linear quadratic control problem" in 2003 [9]. Further development was made in 2017 by A.Ghosh, A.Das and T.E. Aman in their research paper [1]. In [6] S.Islam and A.Das discussed about the properties of bounded operators in $\Gamma$-Hilbert Space. Boundedness of an operator is a great tool to elaborate $\Gamma$-Hilbert Space. We often deal with operators which are not bounded. In this paper, we will briefly discuss the concept, methods and theory of unbounded operators in $\Gamma$-Hilbert Space. In this paper, after consulting the main author, we have made some changes in the main definition of $\Gamma$-Hilbert space [9].

First, we recall the definitions of $\Gamma$-Hilbert Space.

Definition 1.1. Let $E$ be the linear space over the field $F$ and $\Gamma$ be a semi group with respect to addition. A mapping $\langle., . .\rangle:. E \times \Gamma \times E \rightarrow \mathrm{F}(\mathbb{R}$ or $\mathbb{C})$ is called a $\Gamma$-Inner product on $(E, \Gamma)$ if

(i) $\langle., \ldots\rangle$ is linear in first variable and additive in second variable.

(ii) $\langle\mathrm{u}, \gamma, \mathrm{v}\rangle=\langle\mathrm{v}, \gamma, \mathrm{u}\rangle \forall \mathrm{u}, \mathrm{v} \in \mathrm{E}$ and $\gamma \in \Gamma$.

(iii) $\langle\mathrm{u}, \gamma, \mathrm{u}\rangle>0 \forall u \neq 0$.

(iv) $\langle u, \gamma, u\rangle=0$ if at least one of $u, \gamma$ is zero.

\footnotetext{
${ }^{1}$ sahincool92@gmail.com (Corresponding Author); ${ }^{2}$ nrmlsrkr@gmail.com; ${ }^{3}$ ashoke.avik@gmail.com

1,2,3 Department of Mathematics, Raiganj University, West Bengal,India

Article History: Received: 20.04.2021 — Accepted: 09.09.2021 — Published: 10.10.2021
} 
$[(E, \Gamma),\langle., . .\rangle$.$] is called a \Gamma$-inner product space over $F$.

A complete $\Gamma$-inner product space is called $\Gamma$-Hilbert space.

Using the $\Gamma$-inner product, we may define three types of norm in a $\Gamma$-Hilbert space, namely (i) $\gamma$-norm (ii) $\Gamma_{\text {inf }^{-}}$norm and (iii) $\Gamma$-norm.

Definition 1.2. Now if we write $\|u\|_{\gamma}{ }^{2}=\langle u, \gamma, u\rangle$, for $u \in H$ and $\gamma \in \Gamma$ then $\|u\|_{\gamma}{ }^{2}$ satisfy all the conditions of norm.

Definition 1.3. If we define $\|u\|_{\Gamma_{\text {inf }}}=\inf \left\{\|u\|_{\gamma}: \gamma \in \Gamma\right\}$. Clearly $\Gamma_{\text {inf }}$-norm satisfy all the conditions of the norm for $u \in H$.

Definition 1.4. If we write $\|u\|_{\Gamma}=\left\{\|u\|_{\gamma}: \gamma \in \Gamma\right\}$ then this norm is called the $\Gamma$-norm of the $\Gamma$-Hilbert space.

Definition 1.5. Let $\mathrm{L}$ be a non-empty subset of a $\Gamma$-Hilbert space $\mathrm{H}_{\Gamma}$. Two elements $x$ and $y$ are said to be $\gamma$-orthogonal if their inner product $\langle x, \gamma, y\rangle=0$. In symbol, we write $x \perp_{\gamma} y$.

\section{Basic Concepts}

In this section, we briefly discuss about the definition of densely defined operator and the adjoint, selfadjoint, symmetric etc of that operator. Also, related examples and theorem are mentioned in this part.

\subsection{Extension of operators}

Let $\mathrm{S}$ and $\mathrm{T}$ be two operators in a vector space $\mathrm{E}_{\mathrm{S}} \mathrm{D}_{\mathrm{S}}$ and $\mathrm{D}_{\mathrm{T}}$ are the domains of $\mathrm{S}$ and $\mathrm{T}$ respectively. If

$$
\mathrm{D}_{\mathrm{S}} \subset \mathrm{D}_{\mathrm{T}} \text { and } \mathrm{Sx}=\mathrm{Tx} \quad \text { for every } \mathrm{x} \in D_{S}
$$

then $\mathrm{T}$ is called an extension of $\mathrm{S}$ and we write $\mathrm{S} \subset \mathrm{T}$.

\subsection{Densely defined operator}

An operator T defined a linear map T from a subspace of $\mathrm{H}_{\Gamma}$ to $\mathrm{H}_{\Gamma}$ is called an operator in $\mathrm{H}_{\Gamma}$ and the subspace denoted by $\mathrm{D}_{\mathrm{T}}$, is called the domain of $\mathrm{T}$. Now an operator $\mathrm{T}$ is defined in a normed space $\mathrm{E}$ is called densely defined if its domain $\mathrm{D}_{\mathrm{T}}$ is a dense subset of $\mathrm{E}$, that is $\quad \mathrm{cl}_{\mathrm{T}}=\mathrm{E}$.

Example 2.2.1. The differential operator $\frac{d}{d x}$ is densely defined in $L^{2}(\mathbb{R})$, because the subspace of differentiable functions is dense in $L(\mathbb{R})^{2}$.

Theorem 2.2.2. Let $\mathrm{T}$ be a densely defined operator in a $\Gamma$-Hilbert space $\mathrm{H}_{\Gamma}$ and let $\mathrm{E}$ be the set of all $y \in$ $\mathrm{H}_{\Gamma}$ for which $\langle T x, \gamma, x\rangle$ where $\gamma \in \Gamma$ is a continuous functional on $\mathrm{D}_{\mathrm{T}}$. There exists a unique operator $\mathrm{S}$ defined on E such that

$$
\langle T x, \gamma, x\rangle=\langle x, \gamma, S y\rangle \text { for all } x \in \mathrm{D}_{\mathrm{T}} \text { and } \mathrm{y} \in \mathrm{E} .
$$

Proof: For any $\mathrm{y} \in \mathrm{E}$, consider the functional $f_{y}(x)=\langle T x, \gamma, x\rangle$ where $\gamma \in \Gamma$. Being continuous on a dense subspace of $\mathrm{H}_{\Gamma}$, has a unique extension to a continuous functional $\tilde{f}_{y}$ on $\mathrm{H}_{\Gamma}$. 
By Riesz representation theorem, there exists a unique $Z_{y} \in \mathrm{H}_{\Gamma}$ such that $\tilde{f}_{y}(x)=\left\langle x, \gamma, Z_{y}\right\rangle \forall x \in \mathrm{H}_{\Gamma}$. Now if we define $S(y)=Z_{y}$, then we will have

$$
\begin{aligned}
\langle T x, \gamma, x\rangle=f_{y}(x) & =\tilde{f}_{y}(x) \\
= & \left\langle x, \gamma, Z_{y}\right\rangle \\
= & \langle x, \gamma, S y\rangle \text { for all } x \in \mathrm{D}_{\mathrm{T}}, \mathrm{y} \in \mathrm{E} \text { and } \gamma \in \Gamma .
\end{aligned}
$$

Also the linearity of $S$ is obvious.

\subsection{Adjoint of densely defined operator}

Let $\mathrm{T}$ be an operator which is densely defined in a $\Gamma$-Hilbert space $\mathrm{H}_{\Gamma}$. The adjoint $\mathrm{T}^{*}$ of $\mathrm{T}$ is the operator defined on the set of all $y \in \mathrm{H}_{\Gamma}$ for which $\langle T x, \gamma, x\rangle$ where $\gamma \in \Gamma$ is a continuous function on $\mathrm{D}_{\mathrm{T}}$ and such that

$$
\langle T x, \gamma, x\rangle=\left\langle x, \gamma, T^{*} y\right\rangle \text { for all } x \in \mathrm{D}_{\mathrm{T}} \text { and } y \in D_{T^{*}}
$$

Example 2.3.1. Let $C^{1}{ }_{0}(\mathbb{R})$ denote the space of all continuously differentiable functions on $\mathbb{R}$. This is also a dense subspace of $L^{2}(\mathbb{R})$. Now consider the differentiable operator D which defined on $C^{1}{ }_{0}(\mathbb{R})$. Since

$$
\begin{aligned}
\langle D x, \gamma, y\rangle & =\int_{-\infty}^{\infty}\left(\frac{d}{d t} x(t)\right) \gamma \overline{y(t)} d t \\
& =-\int_{-\infty}^{\infty} x(t)\left(\frac{d}{d t} \overline{y(t)}\right) \gamma d t \quad \text { for all } \gamma \in \Gamma .
\end{aligned}
$$

$\therefore\langle D x, \gamma, y\rangle$ is a continuous functional on $C^{1}{ }_{0}(\mathbb{R})$.

Moreover,

$$
\begin{aligned}
\langle D x, \gamma, y\rangle & =-\int_{-\infty}^{\infty} x(t)\left(\frac{d}{d t} \overline{y(t)}\right) \gamma d t . \\
& =\int_{-\infty}^{\infty} x(t) \overline{\left(-\frac{d}{d t}(y(t))\right.} \gamma d t .
\end{aligned}
$$

Here it is not correct to write $D^{*}=-D$, since the domain of $D^{*}$ is not $C^{1}{ }_{0}(\mathbb{R})$.

\subsection{Self -adjoint of densely defined operator}

Let $\mathrm{T}$ be a densely defined operator in a $\Gamma$-Hilbert space $\mathrm{H}_{\Gamma}$. Then $\mathrm{T}$ is called self-adjoint if $T=T^{*}$.

Note. $T=T^{*}$ implies that $D_{T^{*}}=D_{T}$ and $T(x)=T^{*}(x)$ for all $x \in D_{T}$. If $\mathrm{T}$ is a densely defined operator in $\mathrm{H}_{\Gamma}$ which is bounded then $\mathrm{T}$ has a unique extension to a bounded operator in $\mathrm{H}_{\Gamma}$. Then the domain of $\mathrm{T}$ as well as its adjoint $\mathrm{T}^{*}$, is the whole space $\mathrm{H}_{\Gamma}$. If $\mathrm{T}$ is unbounded operators , then $\mathrm{T}$ has an adjoint $\mathrm{T}^{*}$ such that $T(x)=T^{*}(x)$ whenever $x \in D_{T} \cap D_{T^{*}}$, but $D_{T^{*}} \neq D_{T}$ and thus T is not self-adjoint.

\subsection{Symmetric Operator}

We now consider a special kind of operator in $\Gamma$-Hilbert space. An operator $\mathrm{T}$ which is densely defined in $\Gamma$-Hilbert space $\mathrm{H}_{\Gamma}$ is called symmetric if for all $x, y \in D_{T}$, we have

$$
\langle T x, \gamma, y\rangle=\langle x, \gamma, T y\rangle \text { for all } \gamma \in \Gamma \text {. }
$$

It is clear that if $\mathrm{T}$ is symmetric, then $\langle T(x), \gamma, x\rangle \in \mathbb{R}$ for every $x \in D_{T}$ and $\gamma \in \Gamma$. Also, it follows that a densely defined operator $\mathrm{T}$ is symmetric if and only if $\mathrm{T}^{*}$ extends $\mathrm{T}$. If $\mathrm{T}$ is symmetric and $\mathrm{D}_{\mathrm{T}}=\mathrm{H}_{\Gamma}$, then $\mathrm{T}$ is in fact a bounded operator on $\mathrm{H}_{\Gamma}$. This leads as follows,

Let $E=\left\{\mathrm{T}(\mathrm{x}): \mathrm{x} \in \mathrm{H}_{\Gamma},\|\mathrm{x}\|_{\gamma} \leq 1\right\}$. Then for a fixed $y \in \mathrm{H}_{\Gamma}$ and $\gamma \in \Gamma$, we have

$$
\begin{aligned}
|\langle T(x), \gamma, y\rangle| & =|\langle x, \gamma, T(y)\rangle| \\
\leq & \|x\|\|\gamma\|\|T(y)\| \\
& \leq\|T(y)\| \text { for all } x \in \mathrm{H}_{\Gamma} \text { with }\|x\|,\|\gamma\| \leq 1 .
\end{aligned}
$$


Also clearly every self-adjoint operator is symmetric.

Example 2.5.1. Suppose we consider an operator $A=\frac{i d}{d t}$ with the domain $D_{A}=\left\{f \in L^{2}([a, b])\right.$ : $f^{\prime}$ is continuous and $\left.f(a)=f(b)=0\right\}$.

Now, since for all $\gamma \in \Gamma$, we have

$$
\begin{aligned}
\langle A f, \gamma, g\rangle & =\int_{a}^{b} i f^{\prime}(t) \gamma \overline{g(t)} d t \\
& =\int_{a}^{b} f(t) \gamma i \overline{g^{\prime}(t)} d t \\
& =\langle f, \gamma, A g\rangle
\end{aligned}
$$

$\therefore\langle A f, \gamma, g\rangle=\langle f, \gamma, A g\rangle$

for all $f, g \in D_{A}$, A is symmetric.

$\langle A f, \gamma, g\rangle$ is a continuous functional on $D_{A}$ for any function $g$ continuously differentiable, no need to satisfying $g(a)=g(b)$.

Consequently, $D_{A^{*}} \neq D_{A}$ and $\mathrm{A}$ is not self-adjoint.

\subsection{Closed Operator}

A linear operator $T: E_{1} \rightarrow E_{2}$ is said to be closed when the graph $G(T)=\left\{\langle x, \gamma, T x\rangle: x \in D_{T}\right.$ and $\left.\gamma \in \Gamma\right\}$ is a closed subspace of $E_{1} \times E_{2}$ that is

$$
x_{n} \in D_{T}, x_{n} \rightarrow x \text { and } T x_{n} \rightarrow y
$$

implies $x \in D_{T}$ and $T x=y$.

\section{Main Results}

Theorem 3.1. Let $A$ and $B$ be densely defined operators in a $\Gamma$-Hilbert space $\mathrm{H}_{\Gamma}$.

(a) If $A \subset B$, then $B^{*} \subset A^{*}$.

(b) If $D_{B^{*}}$ is dense in $\mathrm{H}_{\Gamma}$, then $B \subset B^{* *}$.

Proof. (a) Let us consider $y \in D_{B^{*}}$ and $\gamma \in \Gamma$. Then as a function of $x,\langle B x, \gamma, y\rangle$ is a continuous functional on $D_{B}$. Also $\langle B x, \gamma, y\rangle$ is a continuous functional on $D_{A}$ since $D_{A} \subset D_{B}$.

Now, $B x=A x$ for $x \in D_{A}$, so $\langle A x, \gamma, y\rangle$ is a continuous functional on $D_{A}$. This proves that $y \in D_{A^{*}}$. Then the equality $A^{*} y=B^{*} y$ for $y \in D_{B^{*}}$ follows from the uniqueness of the adjoint operator.

(b) Let $x \in D_{B}$. Then for every $y \in D_{B^{*}}$ and $\gamma \in \Gamma$, we have

$$
\langle B x, \gamma, y\rangle=\left\langle x, \gamma, B^{*} y\right\rangle
$$

It can be rewrite as

$$
\left\langle B^{*} y, \gamma, x\right\rangle=\langle y, \gamma, B x\rangle .
$$

Since $D_{B^{*}}$ is dense in $\mathrm{H}_{\Gamma}$, $B^{* *}$ exists and we have

$\left\langle B^{*} y, \gamma, x\right\rangle=\left\langle y, \gamma, B^{* *} x\right\rangle$ for all $y \in D_{B^{*}}, x \in D_{B^{* *}}$ and $\gamma \in \Gamma$.

Now, by the proof of (a), we can show that $D_{B} \subset D_{B^{* *}}$ and $B(x)=B^{* *}(x)$ for any $x \in D_{B}$. Thus $B \subset B^{* *}$. 
Theorem 3.2. If $\mathrm{T}$ is a one-to-one operator in a $\Gamma$-Hilbert space and both $\mathrm{T}$ and its inverse $T^{-1}$ are densely defined, then $T^{*}$ is also one- to-one and $\left(T^{*}\right)^{-1}=\left(T^{-1}\right)^{*}$.

Proof. Let $y \in D_{T^{*}}$. Then for every $x \in D_{\mathrm{T}^{-1}}$ and $\gamma \in \Gamma$, we have $T^{-1} x \in D_{T}$ and hence

$$
\begin{gathered}
\left\langle T^{-1} x, \gamma, T^{*} x\right\rangle=\left\langle T T^{-1} x, \gamma, y\right\rangle \\
=\langle x, \gamma, y\rangle .
\end{gathered}
$$

This follows that $T^{*} y \in D_{\left(T^{-1}\right)^{*}}$.

And also,

$$
\left(T^{-1}\right)^{*} T^{*} y=\left(T T^{-1}\right)^{*} y=y
$$

Now we take an arbitrary $y \in D_{\left(T^{-1}\right)^{*}}$.Then for each $x \in D_{T}$ and $\gamma \in \Gamma$, we have

$$
T x \in D_{T^{-1}}
$$

Hence

$$
\left\langle T x, \gamma,\left(T^{-1}\right)^{*} y\right\rangle=\left\langle T^{-1} T x, \gamma, y\right\rangle=\langle x, y\rangle
$$

This shows that $\left(T^{-1}\right)^{*} y \in D_{T^{*}}$. And $T^{*}\left(T^{-1}\right)^{*} y=\left(T^{-1} T\right)^{*} y=y$. Now, from (3.1) and (3.2) it follows that $\left(T^{*}\right)^{-1}=\left(T^{-1}\right)^{*}$.

Theorem 3.3. If $\mathrm{A}, \mathrm{B}$ and $\mathrm{AB}$ are densely defined operators in $\mathrm{H}_{\Gamma}$, then $B^{*} A^{*}=(A B)^{*}$.

Proof. Let $x \in D_{A B}$ and $y \in D_{B^{*}} A^{*}$. Since $x \in D_{B}$ and $A^{*} y \in D_{B^{*}}$, it follows that

$$
\left\langle B x, \gamma, A^{*} y\right\rangle=\left\langle x, \gamma, B^{*} A^{*} y\right\rangle \text { for all } \gamma \in \Gamma .
$$

On the other side, since $B x \in D_{A}$ and $y \in D_{A^{*}}$, we have

$$
\langle A B x, \gamma, y\rangle=\left\langle B x, \gamma, A^{*} y\right\rangle \text { for all } \gamma \in \Gamma .
$$

Hence

$$
\langle A B x, \gamma, y\rangle=\left\langle x, \gamma, B^{*} A^{*} y\right\rangle
$$

Since this holds for all $x \in D_{A B}$, we have $y \in D_{(A B)^{*}}$ and $\left(B^{*} A^{*}\right) y=(A B)^{*} y$. This implies, $B^{*} A^{*}=(A B)^{*}$.

Theorem 3.4. A densely defined operator $T$ in a $\Gamma$-Hilbert space $H_{\Gamma}$ is symmetric if and only if $T=T^{*}$.

Proof: Let us suppose $T=T^{*}$. Since for all $x \in \mathrm{D}_{\mathrm{T}}$ and $y \in D_{T^{*}}$ we have

Again we have

$$
\langle T x, \gamma, y\rangle=\left\langle x, \gamma, T^{*} y\right\rangle \text { where } \gamma \in \Gamma
$$

$$
\langle T x, \gamma, y\rangle=\langle x, \gamma, T y\rangle \text { for all } x, y \in D_{T}
$$

Thus, $\mathrm{T}$ is symmetric. If $\mathrm{T}$ is symmetric then combining (3.3) and (3.4) we can conclude $T=T^{*}$.

Corollary 3.5. If $\mathrm{T}$ is a densely defined symmetric operator, then $\mathrm{T}^{*}$ is the maximal symmetric extension of T. 
Proof. Let $\mathrm{S}$ be a symmetric operator in a $\Gamma$-Hilbert space $\mathrm{H}_{\Gamma}$ such that $\mathrm{T} \subset \mathrm{S}$. Then by the Theorem 3.3, we have

$$
\mathrm{S}^{*} \subset \mathrm{T}^{*}
$$

Hence, $\mathrm{T} \subset \mathrm{S} \subset \mathrm{S}^{*} \subset \mathrm{T}^{*}$.

Theorem 3.6. If $\mathrm{T}$ is closed and invertible, then $T^{-1}$ is closed.

Proof. Let us suppose that graph of T that is $G(T)$ is closed and $G(T)=\left\{(x, \gamma, T x): x \in D_{T}\right.$ and $\left.\gamma \in \Gamma\right\}$. Then obviously

$$
G\left(T^{-1}\right)=\left\{(T x, \gamma, x): x \in D_{T} \text { and } \gamma \in \Gamma\right\} \text { is closed. }
$$

Theorem 3.7. If $\mathrm{T}$ is densely defined operator, then $\mathrm{T}^{*}$ is closed.

Proof: If $y_{n} \in D_{A^{*}}, y_{n} \rightarrow y$ and $A^{*} y_{n} \rightarrow z$, then for any $x \in D_{A} \& \gamma \in \Gamma$ we have

$$
\begin{aligned}
\langle A x, \gamma, y\rangle & =\lim _{n \rightarrow \infty}\left\langle A x, \gamma, y_{n}\right\rangle \\
& =\lim _{n \rightarrow \infty}\left\langle x, \gamma, A^{*} y_{n}\right\rangle \\
& =\langle x, \gamma, z\rangle
\end{aligned}
$$

Hence, $y \in D_{A^{*}}$ and $A^{*} y=z$.

Note. If the given operator $\mathrm{A}$ is not closed then is it possible to extend $\mathrm{A}$ to a closed operator? Answer to that problem is to use the closure of $G(A)$ in $\mathrm{H}_{\Gamma} \times \mathrm{H}_{\Gamma}$ to define an operator. If closure of $G(A)$ defines an operator, then extension of $\mathrm{A}$ is closed.

Theorem 3.8. Every symmetric and densely defined operator in $\Gamma$-Hilbert space has a closed symmetric extension.

Proof. Let A be a densely defined, symmetric operator in a $\Gamma$-Hilbert space $\mathrm{H}_{\Gamma}$. At first, we will show that condition $x_{n} \in D_{A}, x_{n} \rightarrow 0$, as $A x_{n} \rightarrow y$ which implies that $y=0$, is satisfied.

Let $x_{n} \rightarrow 0$ and $A x_{n} \rightarrow y$. Since A is symmetric then for all $\gamma \in \Gamma$ we have

$$
\begin{aligned}
\langle y, \gamma, z\rangle & =\lim _{n \rightarrow 0}\left\langle A x_{n}, \gamma, z\right\rangle \\
& =\lim _{n \rightarrow 0}\left\langle x_{n}, \gamma, A z\right\rangle \\
& =0, \quad \text { for any } z \in D_{A} .
\end{aligned}
$$

This implies $y=0$, as $D_{A}$ is dense in $\mathrm{H}_{\Gamma}$.

Now we have that there exists a closed operator B such that $G(B)=\operatorname{Cl} G(A)$ and hence $\mathrm{A} \subset \mathrm{B}$. We have to prove that B is symmetric. If $x, y \in D_{B}$, then there exists $x_{n}, y_{n} \in D_{A}$ such that

$$
x_{n} \rightarrow x \quad, \quad A x_{n} \rightarrow A x
$$

and

$$
y_{n} \rightarrow y \quad, \quad B x_{n} \rightarrow B x
$$

Since A is a symmetric operator, we have

$$
\left\langle A x_{n}, \gamma, y_{n}\right\rangle=\left\langle x_{n}, \gamma, A y_{n}\right\rangle \text { for all } \gamma \in \Gamma .
$$


Then by letting $n \rightarrow \infty$, we have

$$
\langle B x, \gamma, y\rangle=\langle x, \gamma, B y\rangle
$$

Hence B is symmetric.

Theorem 3.9. Let $\mathrm{T}$ be a closed densely defined operator in a $\Gamma$-Hilbert space $H_{\Gamma}$. Then

(a) For any $v, w \in \mathrm{H}_{\Gamma}$, there exist unique $x \in D_{T}$ and $y \in D_{T^{*}}$ such that $T(x)+y=v$ and $x-T^{*}(y)=$ $w$.

(b) For any $w \in \mathrm{H}_{\Gamma}$, there exist unique $x \in D_{T^{*} T}$ such that $x+T^{*} T(x)=w$.

Proof. (a) Consider the $\Gamma$-Hilbert space $\mathrm{H}_{\Gamma_{1}}=\mathrm{H}_{\Gamma} \times \mathrm{H}_{\Gamma}$. Since $\mathrm{T}$ is closed, $G(T)=\{(x, \gamma, T(x)): x \in$ $D_{T}$ and $\left.\gamma \in \Gamma\right\}$ is a closed subspace of $\mathrm{H}_{\Gamma_{1}}$. Then by the projection theorem we have

$$
\mathrm{H}_{\Gamma_{1}}=\mathrm{G}(\mathrm{T})+\mathrm{G}(\mathrm{T})^{\perp_{\gamma}}
$$

with

$$
\mathrm{G}(\mathrm{T}) \cap \mathrm{G}(\mathrm{T})^{\perp_{\gamma}}=\{0\}
$$

Now, $(u, y) \in \mathrm{G}(\mathrm{T})^{\perp_{\gamma}}$ if and only if $\langle(x, T x), \gamma,(u, y)\rangle=0$ for all $x \in D_{T}$ and $\gamma \in \Gamma$. This implies, $\langle x, \gamma, u\rangle+\langle T(x), \gamma, y\rangle=0$. That is $(u, y) \in \mathrm{G}(\mathrm{T})^{\perp_{\gamma}}$ if and only if $\langle T(x), \gamma, y\rangle=\langle x, \gamma,-u\rangle$ for all $x \in D_{T}$. In other way,

$$
(u, y) \in \mathrm{G}(\mathrm{T})^{\perp_{\gamma}} \text { if and only if } y \in D_{T^{*}} \text { and } u=-T^{*}(y) .
$$

Since $(w, v) \in \mathrm{H}_{\Gamma} \times \mathrm{H}_{\Gamma}$, then there exist unique $x \in D_{T}$ and $y \in D_{T^{*}}$ such that

$$
(w, \gamma, v)=(x, \gamma, T(x))+\left(-T^{*}(y), \gamma, y\right) \text { for all } \gamma \in \Gamma .
$$

That is, $w=x-T^{*}(y)$ and $v=T(x)+y$.

(b) Letting $v=0$ in (a), then there exist unique $x \in D_{T}$ and $y \in D_{T^{*}}$ such that $T(x)+y=0$ and $x-$ $T^{*}(y)=w$. Thus $x-T^{*}(-T(x))=0$ implies, $x+T^{*} T(x)=w$, as desired.

\section{References}

[1] A. Ghosh, A. Das, T.E. Aman, Representation theorem on $\Gamma$-Hilbert space, International J. of Math Trends and Tech.,52(9), (2017) 608-615.

[2] B.K. Lahiri, Elements of functional analysis, The World Press Private Limited, (1982), Kolkata.

[3] B. V. Limaye, Functional Analysis, New age International (p) Limited, Publishers, Delhi, (2013), India.

[4] E. Kreyszig, Introductory functional analysis with applications, (1978), New York: Wiley.

[5] M.T. Garayev, M. Gürdal, S. Saltan, Hardy type inequaltiy for reproducing kernel Hilbert space operators and related problems, Positivity, 21(4), (2017), 1615-1623.

[6] S. I. Islam, On Some bounded Operators and their characterizations in $\Gamma$-Hilbert Space, Cumhuriyet Science Journal, 41(4), (2020), 854-861.

[7] J. B. Conway, A course in functional analysis (Vol. 96), (2019), Springer. 
[8] L. Debnath, P. Mikusinski, Introduction to Hilbert spaces with applications, (2005), Academic press.

[9] T.E. Aman, D. K. Bhattacharya, T-Hilbert space and linear quadratic control problem, Revista de la Academia Canaria de Ciencias:= Folia Canariensis Academiae Scientiarum, 15(1), (2003), 107-114. 